\title{
Numerical Study on Hydrogen Flow Behavior in Two Compartments with Different Connecting Pipes
}

\author{
HanChen Liu and XueWu Cao \\ School of Mechanical Engineering, Shanghai Jiao Tong University, 800 Dong Chuan Rd., Shanghai 200240, China \\ Correspondence should be addressed to XueWu Cao; caoxuewu@situ.edu.cn
}

Received 9 October 2017; Accepted 23 November 2017; Published 14 December 2017

Academic Editor: Eugenijus Ušpuras

Copyright (C) 2017 HanChen Liu and XueWu Cao. This is an open access article distributed under the Creative Commons Attribution License, which permits unrestricted use, distribution, and reproduction in any medium, provided the original work is properly cited.

\begin{abstract}
Hydrogen accumulation in the containment compartments under severe accidents would result in high concentration, which could lead to hydrogen deflagration or detonation. Therefore, getting detailed hydrogen flow and distribution is a key issue to arrange hydrogen removal equipment in the containment compartments. In this study, hydrogen flow behavior in local compartments has been investigated in two horizontal compartments. The analysis model is built by 3-dimensional CFD code in Cartesian coordinates based on the connection structure of the Advanced Pressurized Water Reactor (PWR) compartments. It consists of two cylindrical vessels, representing the Steam Generator compartment (SG) and Core Makeup Tank compartment (CMT). With standard $k-\varepsilon$ turbulence model, the effects of the connecting pipe size and location on hydrogen concentration distribution are investigated. Results show that increasing the diameter of connection pipe (IP) which is located at $800 \mathrm{~mm}$ from $150 \mathrm{~mm}$ to $300 \mathrm{~mm}$ facilitates hydrogen flow between compartments. Decreasing the length of IP which is located at $800 \mathrm{~mm}$ from $1000 \mathrm{~mm}$ to $500 \mathrm{~mm}$ can also facilitate hydrogen flow between compartments. Lower IP is in favor of hydrogen mixing with air in non-source compartment. Higher IP is helpful for hydrogen flow to the non-source term compartment from source term compartment.
\end{abstract}

\section{Introduction}

After the Fukushima nuclear accident in 2011, hydrogen risk and control issues in severe accidents of Nuclear Power Plants (NPPs) became the key problem of the industry. In the course of a hypothetical severe accident in NPPs, hydrogen is generated in primary loop by the reaction of steam and fuelcladding and released with steam into the containment and compartments from the break location [1]. Such generated hydrogen is invariably transported into the compartments.

Hydrogen distribution can be influenced by the complex channel structures inside the containment and the break location, which results in uniform hydrogen concentration. High local hydrogen concentrations may occur and create favorable conditions for combustion or detonation, which is a great threat to the integrity of the containment [2]. Moreover even local hydrogen burning, which cannot be a threat to containment integrity, may also be hazardous to the survivability of some important equipment or instruments. In TMI accident as a real example, $500 \mathrm{~kg}$ of hydrogen was released and 27 psig of peak pressure caused by $\mathrm{H} 2$ burning was measured in the containment [3]. In order to reduce the potential risk of hydrogen, the distribution, mixing, and stratification of light gases should be analyzed and understood firstly.

As for containment atmosphere modeling, LumpedParameter (LP) codes and Computational Fluid Dynamics (CFD) codes are used. In recent years, CFD method has become a choice to analyze the hydrogen risk [4]. Threedimensional transport equations of fluid mechanics can be numerically solved by finite volume method or finite element method in codes. Up till now, many CFD analytical works for hydrogen management have been conducted to investigate the hydrogen behavior in the containment. Cheng and Cao [5] used CFD mode to study the hydrogen distribution in the containment under different hydrogen production rates. Visser et al. [6] carried out the validation of FLUENT model for hydrogen distribution in the containment using THAI HM2 test. Royl et al. [7] used GASFLOW code to analyze the steam and hydrogen distribution with passive 


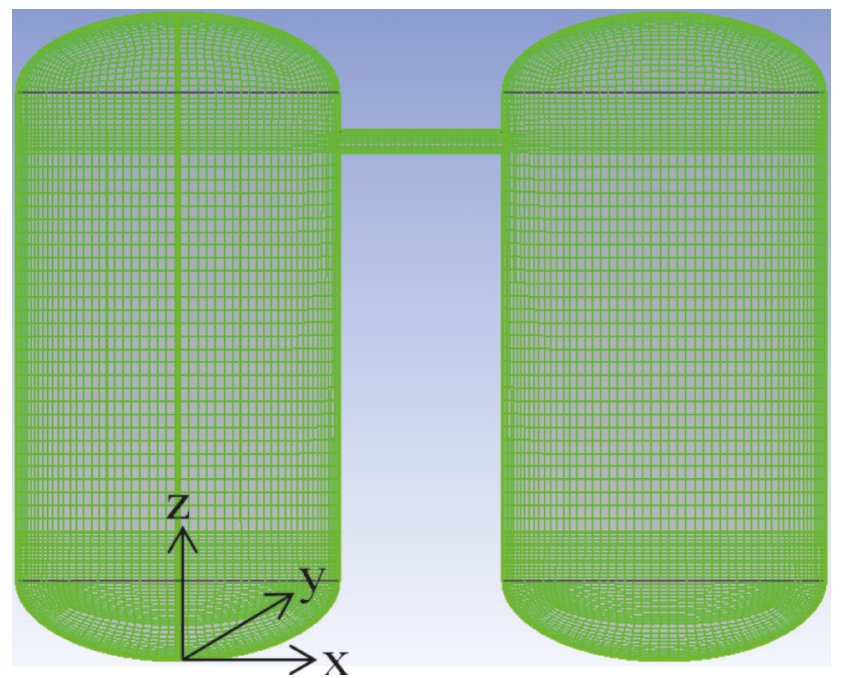

(a) Analysis model and mesh

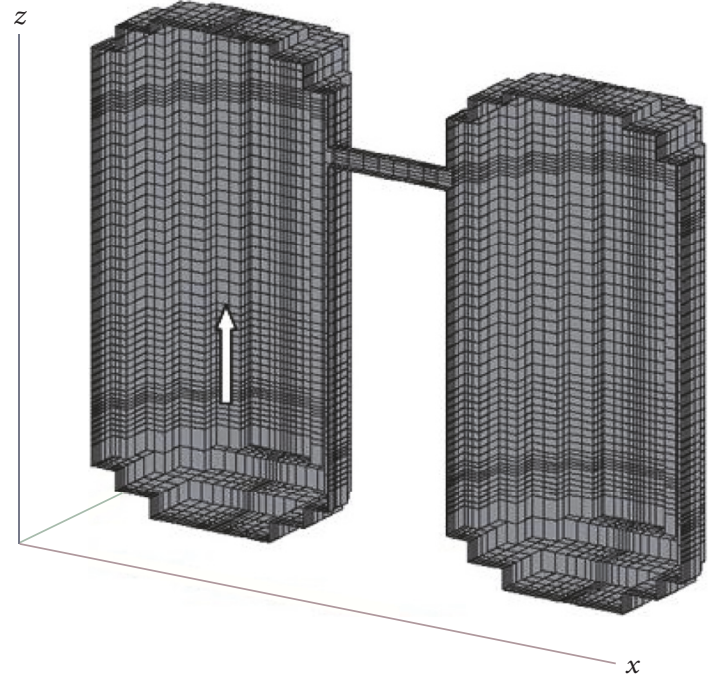

(b) Compared model with GASFLOW

Figure 1: Geometric model built by both codes.

autocatalytic recombiners mitigation in German Konvoi-type NPP. GASFLOW is used to analyze hydrogen distribution for APR1400 containment under hypothetical LOCA [8] and LOFW accident [9]. Previous research shows that CFD method has been adopted to simulate hydrogen distribution in different scale geometric construction and has been verified with experimental data.

In this paper, the hydrogen distribution in two compartments interconnected with different pipe is analyzed to investigate the hydrogen migration and distribution characteristics. The analysis model is built by 3 -dimensional CFD code in Cartesian coordinates based on the connection structure of the Advanced Pressurized Water Reactor (PWR) compartments. It consists of two cylindrical vessels, representing the Steam Generator compartment (SG) and Core Makeup Tank compartment (CMT).

\section{Validation of the Analysis Model}

The analysis model built in this paper is validated by the same model built with GASFLOW, which has been successfully validated in a large number of benchmark exercises, for example, the ISP-47 [10]. Therefore, the effectiveness of the analysis model is analyzed by comparing the simulation results obtained by both codes.

2.1. Geometrical Modeling. Figure 1 shows the geometrical model built in Cartesian coordinates and mesh division. The model consists of interconnected compartments with the pipe located at elevation $3200 \mathrm{~mm}$, and the origin of coordinates is located in the bottom center of the left vessel. Each cylindrical vessel has a height of $4 \mathrm{~m}$, a diameter of $2 \mathrm{~m}$, and inner volume of $11.5 \mathrm{~m}^{3}$. To make the shape and volume of local compartment as accurate as possible, the geometrical model is built in 3D Cartesian structured meshes with 224,528 cells in analysis model and with 95,700 cells for the model built with GASFLOW.
2.2. Turbulence Model. Turbulence is crucial to hydrogen flow distribution. In simulation, appropriate choice of turbulence model has significant effect on hydrogen distribution in local compartments. The standard $k-\varepsilon$ model took into account the full buoyancy effects which is treated as a useful model for plum simulation. Wang and Cao [11] carried out the validation of $k-\varepsilon$ model for hydrogen distribution in the containment using THAI HM2 test. Hou et al. [12] used $k-\varepsilon$ model to study the hydrogen distribution in the containment of Large-Scale Gas Mixing Facility (LSGMF); compared with the experiment data, it is found that the distribution of hydrogen concentration when adopting the standard $k-\varepsilon$ turbulence model is closest to the experiment results. Based on the existing research result about turbulence model, $k-\varepsilon$ turbulence model is used in this simulation.

The $k-\varepsilon$ models belong to the class of two-equation RANS turbulence models. In the $k-\varepsilon$ models, turbulence is modeled with the transport equation for the turbulent kinetic energy $(k)$ and its dissipation rate $(\varepsilon)[6]$.

Reynolds stress is calculated based on Boussinesq hypothesis by obtaining eddy viscosity in $k-\varepsilon$ turbulence model. In $k-\varepsilon$ model, dynamic turbulent viscosity is shown as follows:

$$
\mu_{t}=\frac{\rho C_{\mu} k^{2}}{\varepsilon},
$$

where $\rho$ is density, $C_{\mu}$ is constant $0.05, k$ is turbulence kinetic energy, and $\varepsilon$ is turbulent dissipation rate [13].

The values of $k$ and $\varepsilon$ come directly from the differential transport equations for the turbulence kinetic energy and turbulence dissipation rate:

$$
\begin{aligned}
& \frac{\partial(\rho k)}{\partial t}+\frac{\partial}{\partial x_{j}}\left(\rho U_{j} k\right) \\
& =\frac{\partial}{\partial x_{j}}\left[\left(\mu+\frac{\mu_{t}}{\sigma_{k}}\right) \frac{\partial k}{\partial x_{j}}\right]+P_{k}-\rho \varepsilon+P_{k b}
\end{aligned}
$$



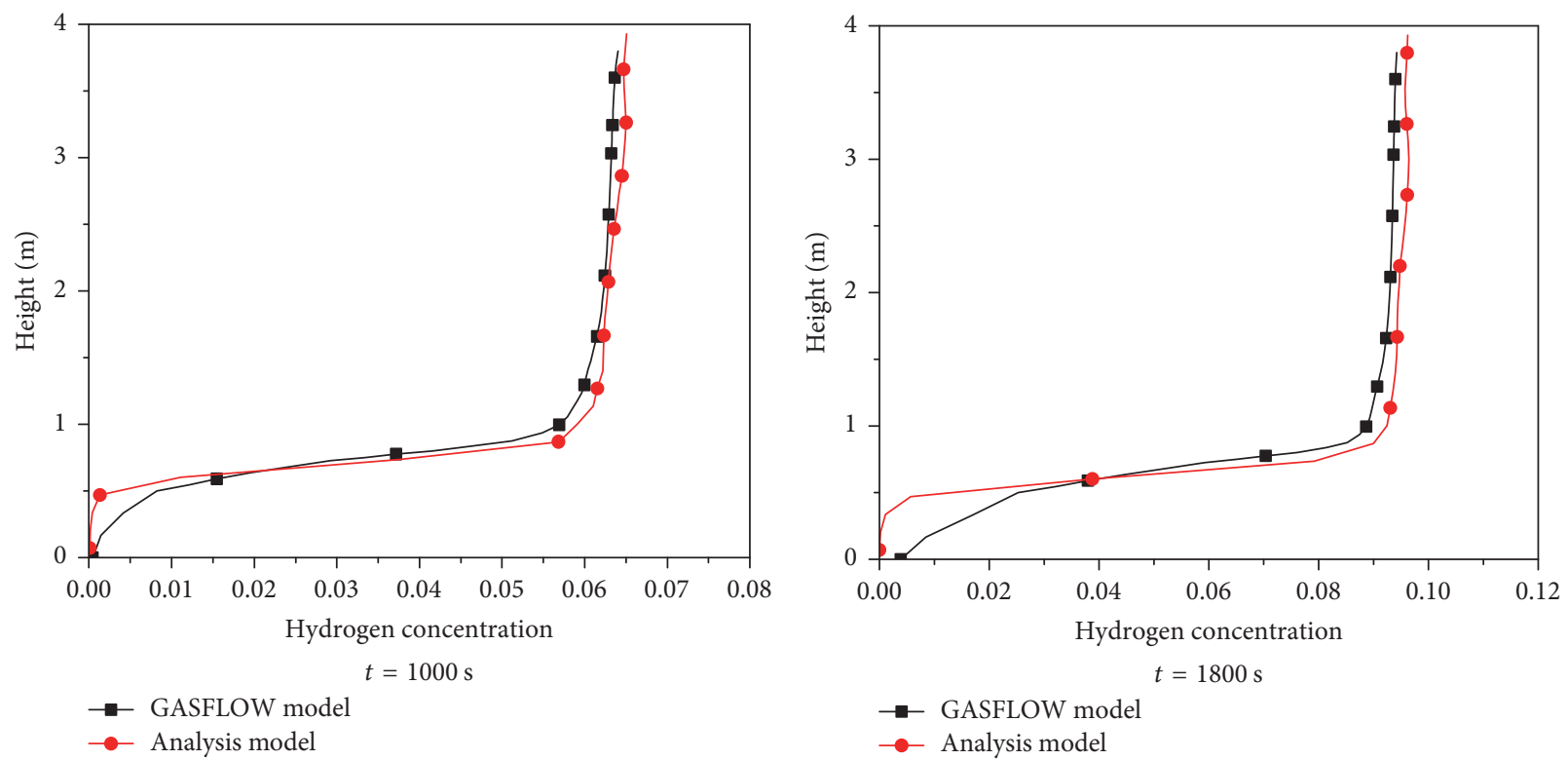

FIGURE 2: Hydrogen concentration distribution in source term compartment.

$$
\begin{aligned}
& \frac{\partial(\rho \varepsilon)}{\partial t}+\frac{\partial}{\partial x_{j}}\left(\rho U_{j} \varepsilon\right) \\
& =\frac{\partial}{\partial x_{j}}\left[\left(\mu+\frac{\mu_{t}}{\sigma_{\varepsilon}}\right) \frac{\partial \varepsilon}{\partial x_{j}}\right] \\
& +\frac{\varepsilon}{k}\left(C_{\varepsilon 1} P_{k}-C_{\varepsilon 2} \rho \varepsilon+C_{\varepsilon 1} P_{\varepsilon b}\right),
\end{aligned}
$$

where $U_{j}$ represents velocity, $t$ represents time, $C_{\varepsilon 1}$ is constant $1.44, C_{\varepsilon 2}$ is constant $1.92, \sigma_{k}$ is constant $1.0, \sigma_{\varepsilon}$ is constant 1.3 , $\mu$ is the molecular viscosity, and $\mu_{t}$ is the turbulence viscosity. $P_{k b}$ and $P_{\varepsilon b}$ represent the influence of the buoyancy forces. $P_{k}$ is the turbulence production due to viscous forces.

2.3. Validation Results. Hydrogen is vertically injected from the central position at the elevation of $800 \mathrm{~mm}$ from the vessel bottom. The mass flow rate of hydrogen released from injection pipe is $0.1 \mathrm{~g} / \mathrm{s}$. The diameter of injection pipe is $30 \mathrm{~mm}$. At the initial time $(t=0 \mathrm{~s})$, air in the multiple compartments is $1 \mathrm{~atm}$. Two measure lines, named measure line 1 and line 2 , are set to measure the hydrogen concentration in both compartments. In the process of modeling, the coordinate origin is located at the bottom center of the source term compartment, as shown in Figure 1. Line 1 is located at the source term compartment, the coordinates of the starting point are $x=0.5 \mathrm{~m}, y=0 \mathrm{~m}$, and $z=0.07 \mathrm{~m}$, and the endpoint coordinates are $x=0.5 \mathrm{~m}, y=0 \mathrm{~m}$, and $z=3.93 \mathrm{~m}$. Line 2 is located at central line in non-source term compartment.

Figure 2 shows hydrogen concentration distribution in source term compartment. The result shows that the hydrogen concentration is in agreement with the two compared models. However, the results are different below the injection area with the hydrogen concentration difference value of no more than 0.01 , showing that hydrogen dispersion is strong under the GASFLOW model, while the hydrogen concentrations in the upper space of the two compartments are in good agreement with each other. Figure 3 shows hydrogen concentration distribution in non-source term compartment. Result shows that the hydrogen concentration predicted with analysis model is slightly lower than that of GASFLOW model at the time of $1000 \mathrm{~s}$ in the whole non-source term compartment, which means less hydrogen transfers into the non-source term compartment at that time in analysis model, while, at the time of $1800 \mathrm{~s}$, the hydrogen concentration reaches the same value in the upper space. Therefore, the analysis model can be used to simulate hydrogen flow behaviors.

\section{Hydrogen Flow Behavior in Two Compartments}

3.1. Cases Selection. Considering the effect factors on hydrogen flow behavior, the analysis cases are selected with different interconnection pipe (IP) diameters, lengths, and locations, shown in Figure 4. In case 1, the pipe diameter is $150 \mathrm{~mm}$ and the length is $1000 \mathrm{~mm}$. In case 2, the pipe diameter is $150 \mathrm{~mm}$ and the length is $500 \mathrm{~mm}$. In case 3, the pipe diameter is $300 \mathrm{~mm}$ and the length is $1000 \mathrm{~mm}$. In case 4 , the pipe diameter is $150 \mathrm{~mm}$ and the length is $1000 \mathrm{~mm}$. In cases 1,2 , and 3, the IP is located at $800 \mathrm{~mm}$. In case 4 , the IP is located at $3200 \mathrm{~mm}$. In all cases, hydrogen is horizontally injected from the side of the source term compartment bottom at the elevation of $800 \mathrm{~mm}$. For each case, two measure lines are named, in which line 1 is located at central line in source term compartment and line 2 is located at central line in non-source term compartment.

3.2. Sensitivity Analyses. Mesh sensitivity study and influence of the time step should be investigated before simulation. The 

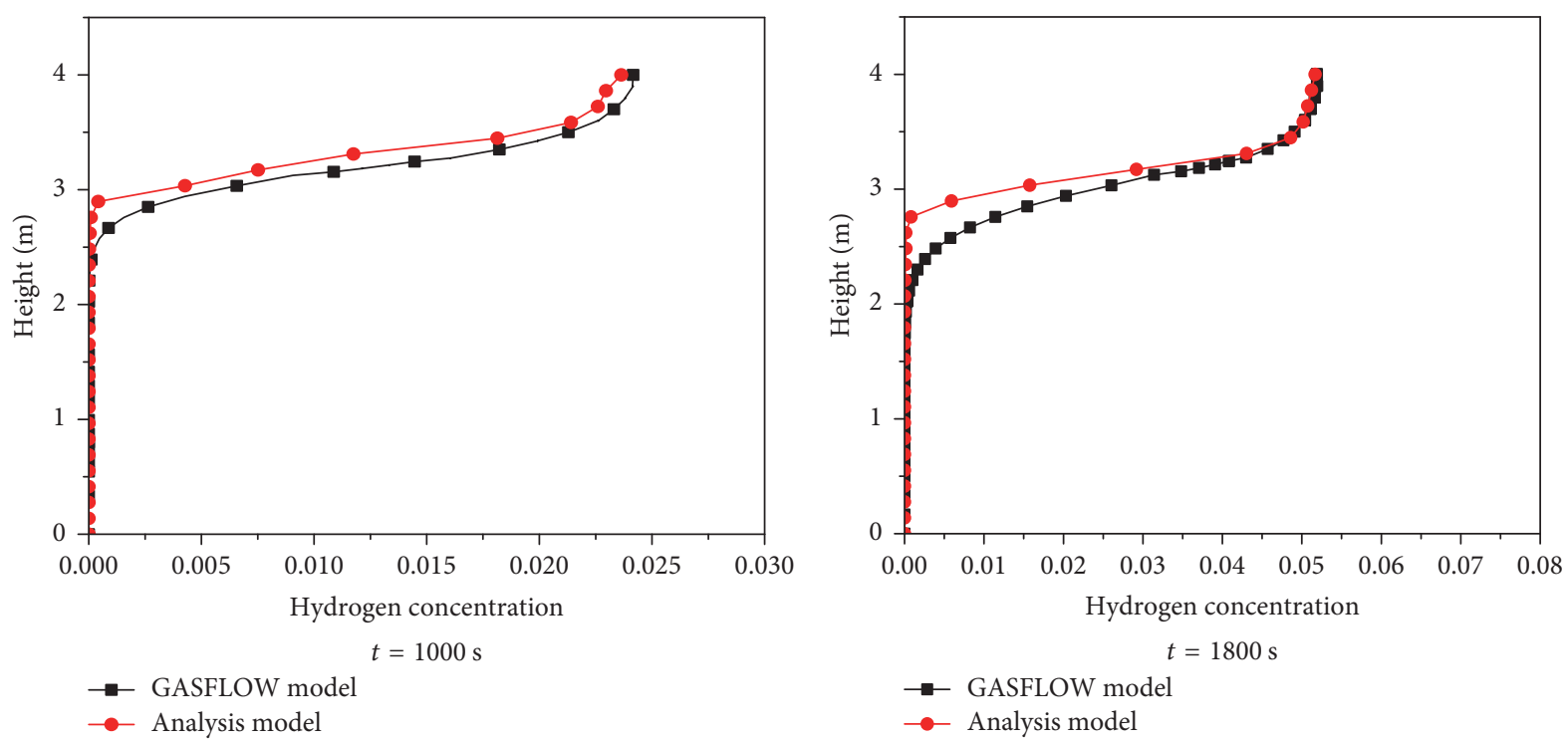

FIGURE 3: Hydrogen concentration distribution in non-source term compartment.

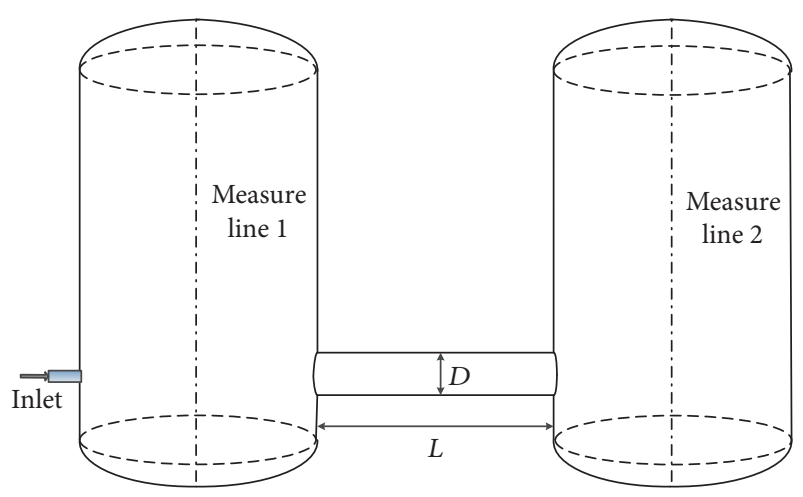

Model of case 1

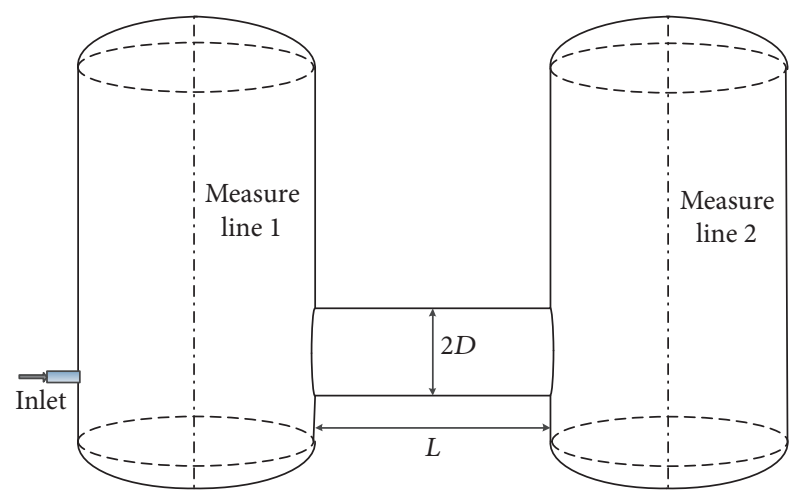

Model of case 3

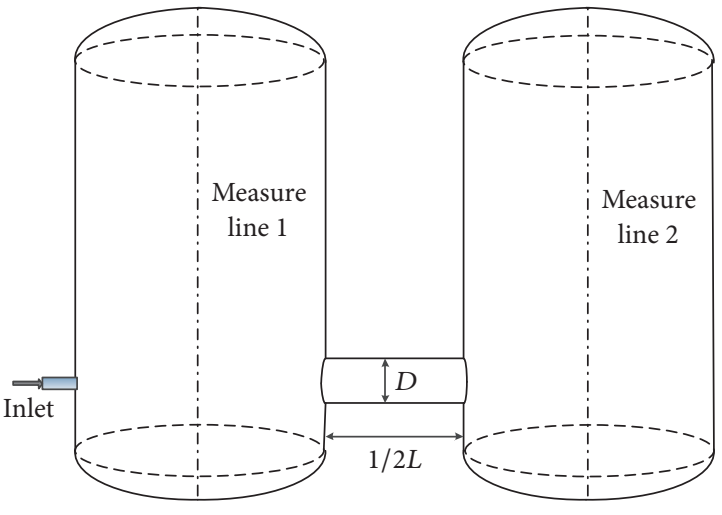

Model of case 2

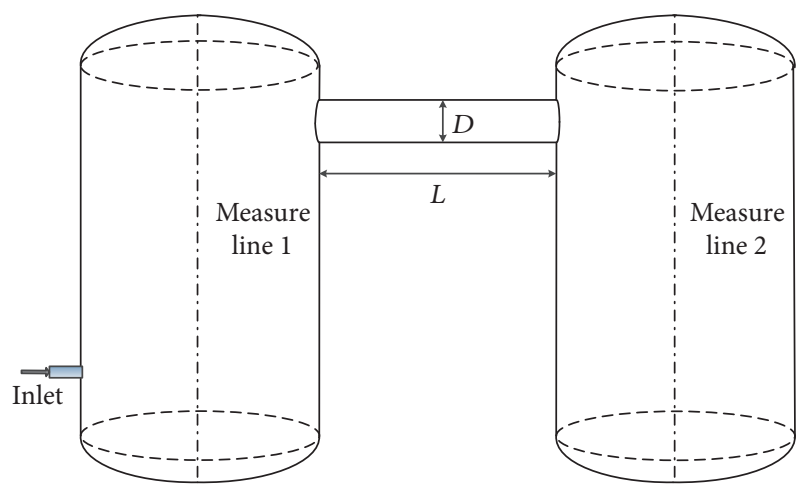

Model of case 4

FIGURE 4: Geometric model of two compartments.

sensitivity analysis is given under case 1 , which is similar to the other cases.

Three kinds of structure meshes are in the range from a few tens of thousands of cells to a few hundreds of thousands to evaluate the grid independence of the solution. The fluid domain is discretized in about 85,735 cells, 219,692 cells, and 415,335 cells, respectively, to simulate the hydrogen concentration vertical distribution in line 1 with standard $k-\varepsilon$ model and the results are summarized in Figure 5. Comparing the results of fine mesh and medium mesh, 

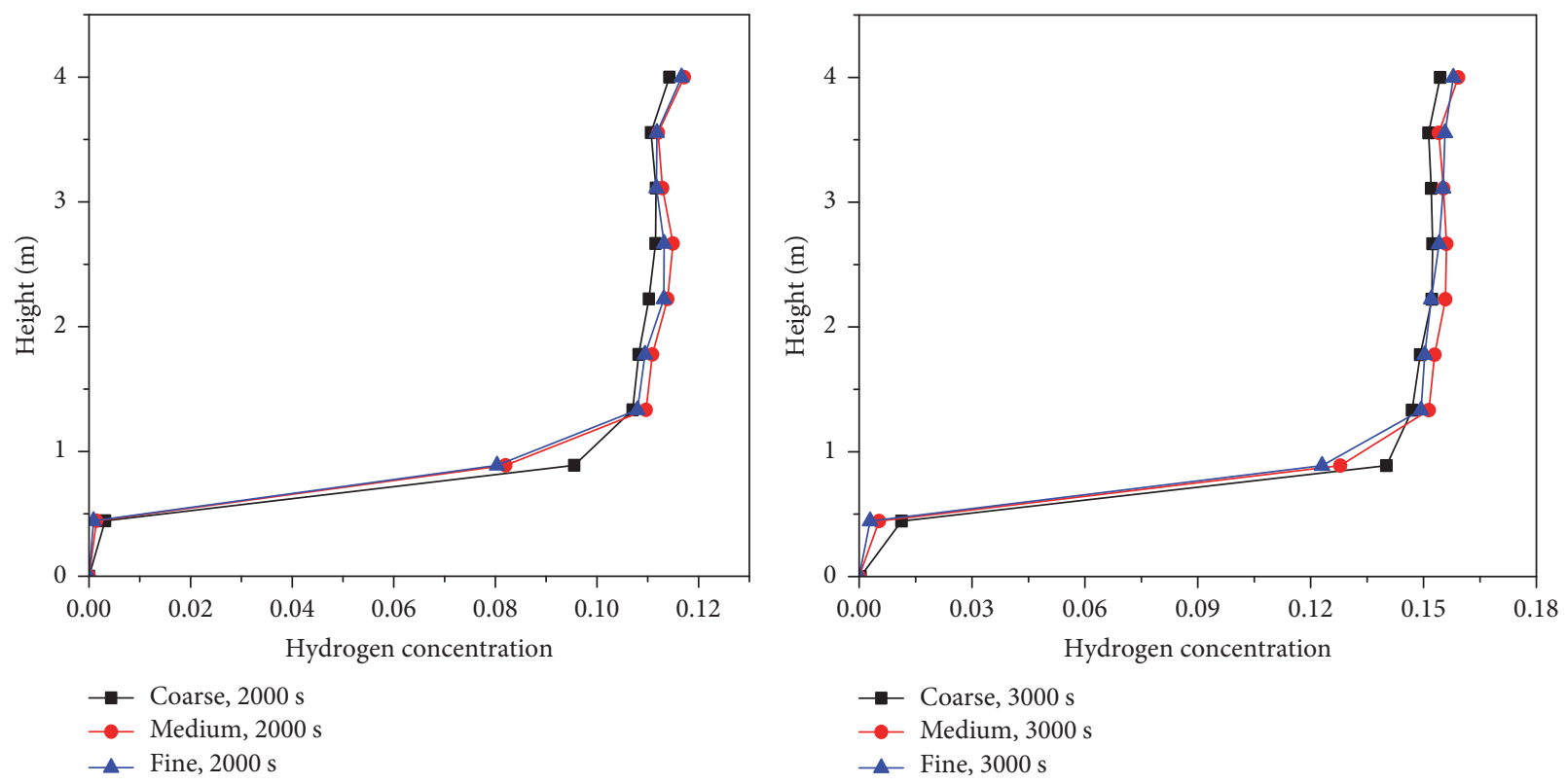

FIGURE 5: Hydrogen distribution for mesh sensitivity on line 1 in source term compartment of case 1.

hydrogen concentrations are similar to each other. The little difference of hydrogen concentration is in the upper space of source term compartment, while the hydrogen concentration predicted with coarse mesh is lower than that of the other mesh. Considering the computational time and accuracy, the medium mesh can be selected to simulate the hydrogen transport in the next study.

Different time steps of $0.01 \mathrm{~s}, 0.03 \mathrm{~s}, 0.05 \mathrm{~s}$, and $0.10 \mathrm{~s}$ are used to simulate the hydrogen concentration by standard $k-\varepsilon$ model. Hydrogen concentration distribution on line 1 at $500 \mathrm{~s}$ is shown in Figure 6, which shows that the hydrogen concentrations with different time steps are in agreement. With the time step size increasing from $0.01 \mathrm{~s}$ to $0.05 \mathrm{~s}$, the calculation time is decreased. With the time step size increasing from $0.05 \mathrm{~s}$ to $0.10 \mathrm{~s}$, due to the increase of iterations number, the calculation time is increased. When the time step size exceeds about $0.10 \mathrm{~s}$, the computational stability is affected and it is hard to converge. Therefore, the time step $0.05 \mathrm{~s}$ is used in the next study.

3.3. Changed IP Size. Hydrogen concentration in source term compartment is higher than that in non-source compartment for cases 1, 2, and 3, as shown in Figure 7. Hydrogen flow behavior in source term compartment is similar due to the same injection mass flow rate and injection pipe diameter. Hydrogen flows upward along the wall from the injection and transports into the upper space. When the upstream gas is resisted by the dome, hydrogen flow direction will be changed, starting to flow around along the wall of the dome and then fill the whole upper space gradually. Hydrogen concentration stratification is established due to the effect of buoyancy. With continuous hydrogen injection, hydrogen distribution in source term compartment is homogeneous gradually due to the effect of diffusion. Hydrogen starts to diffuse into noncompartment when hydrogen flows to the

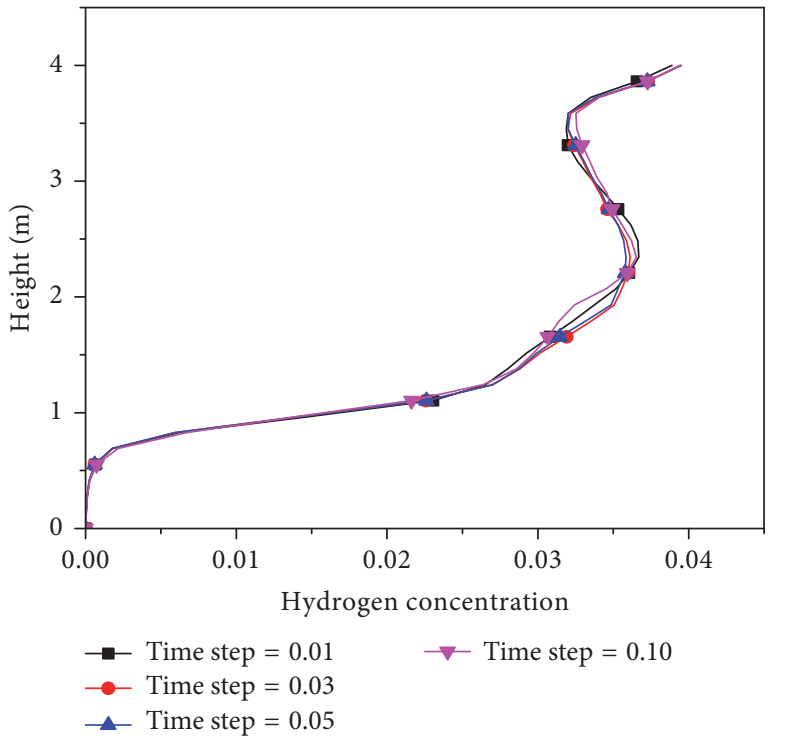

FIGURE 6: Hydrogen concentration for different time steps on line 1 in source term compartment of case 1 .

entrance of the IP at the source term compartment with the similar flow phenomenon due to the lower position of the IP [14].

Figure 8 shows the velocity vector in the IP for case 1 . A counterflow is observed in the IP. The gas mixture leaves source term compartment through the top of the IP to nonsource term compartment where it moves upwards under the effect of buoyancy due to the effect of gas density difference in the two compartments. Air in non-source term compartment is transported with a reversed flow through the IP [14].

Figure 9 shows the quantitative comparison of the dispersion process, showing the change in hydrogen concentration 
He ideal gas, molar fraction

Contour 1

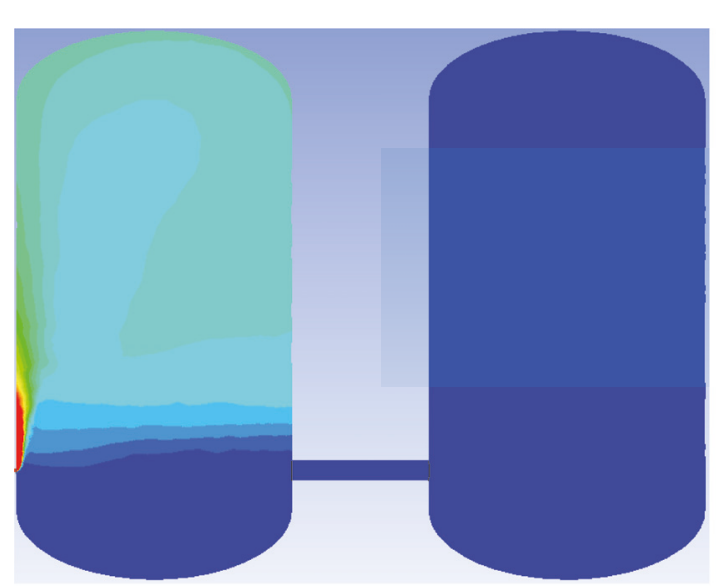

Case $1, t=400 \mathrm{~s}$

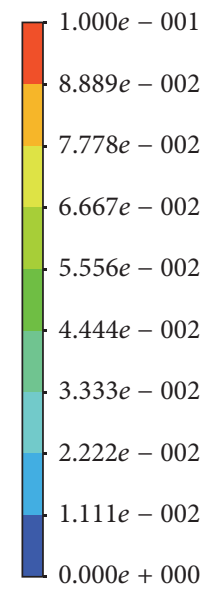

He ideal gas, molar fraction Contour 1

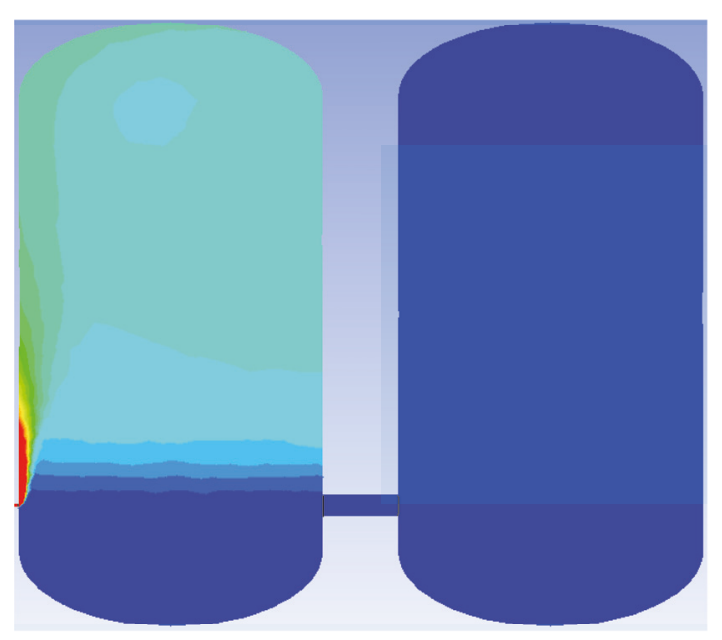

Case $2, t=400 \mathrm{~s}$

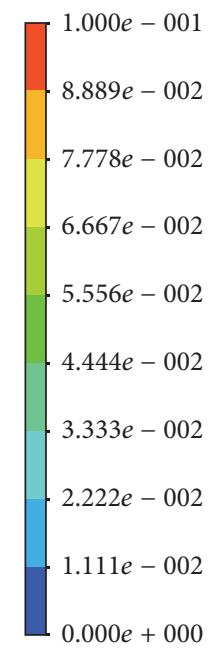

He ideal gas, molar fraction

Contour 1

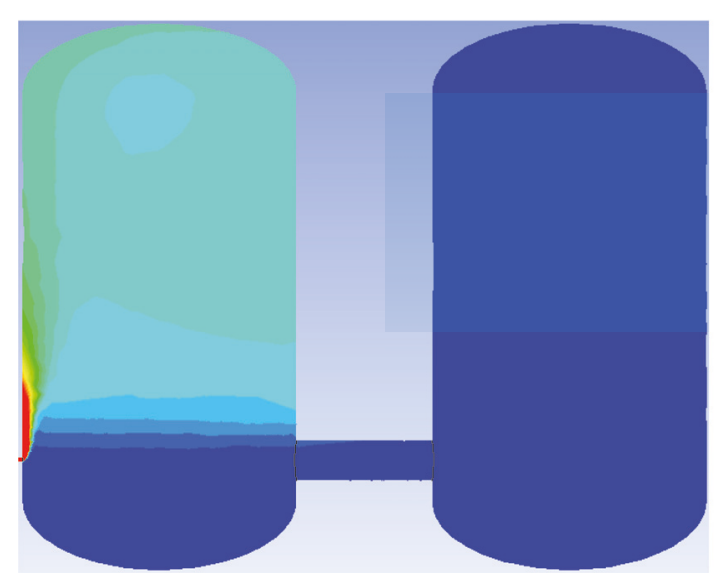

Case $3, t=400 \mathrm{~s}$

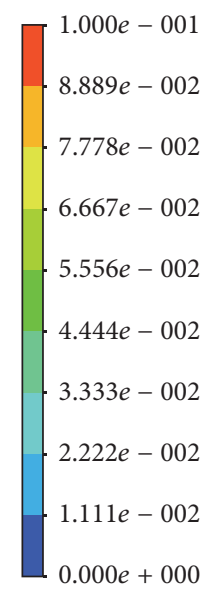

$0.000 e+000$

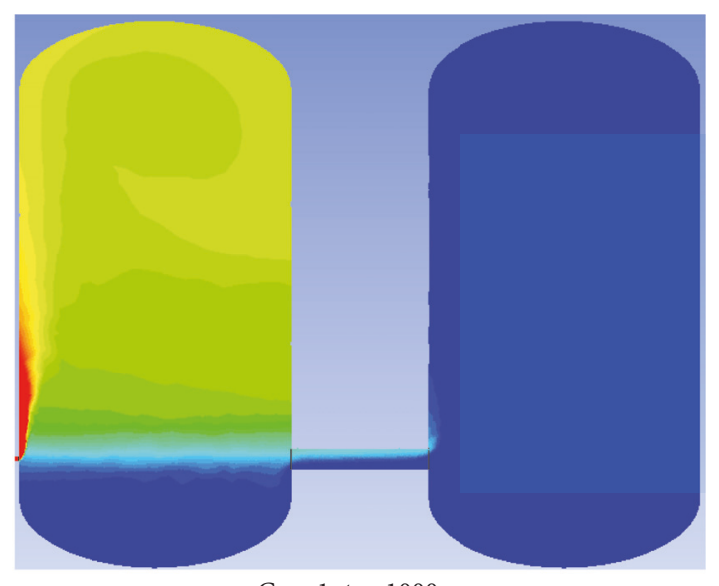

Case $1, t=1000 \mathrm{~s}$

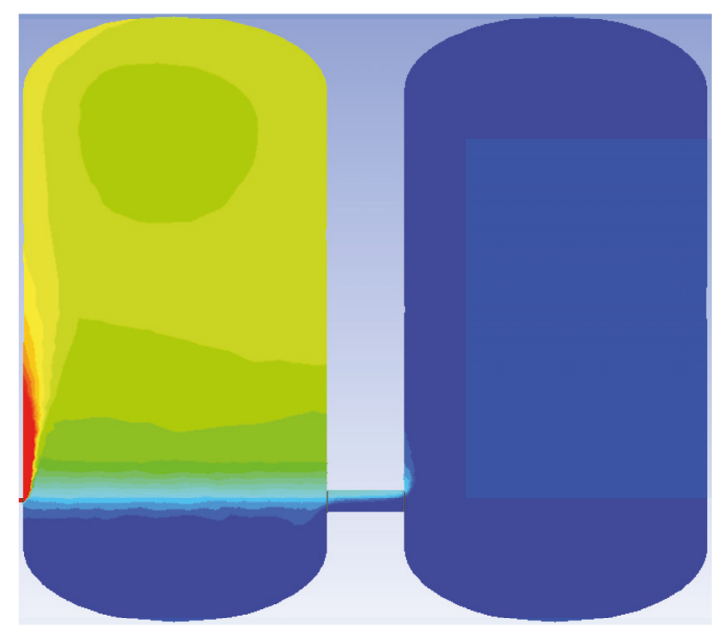

Case 2, $t=1000 \mathrm{~s}$

FIGURE 7: Hydrogen concentration at times $400 \mathrm{~s}$ and $1000 \mathrm{~s}$ in different cases. 


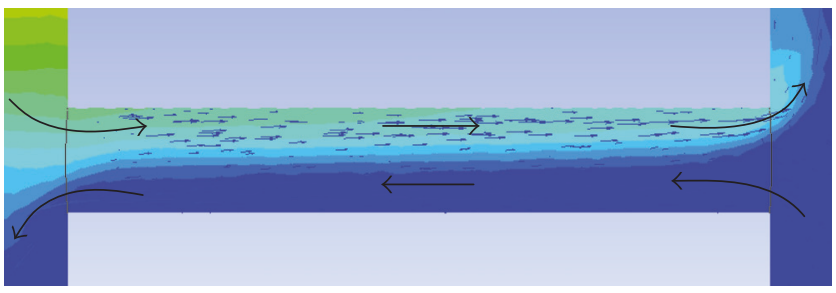

FIGURE 8: Velocity vector in IP in case 1.

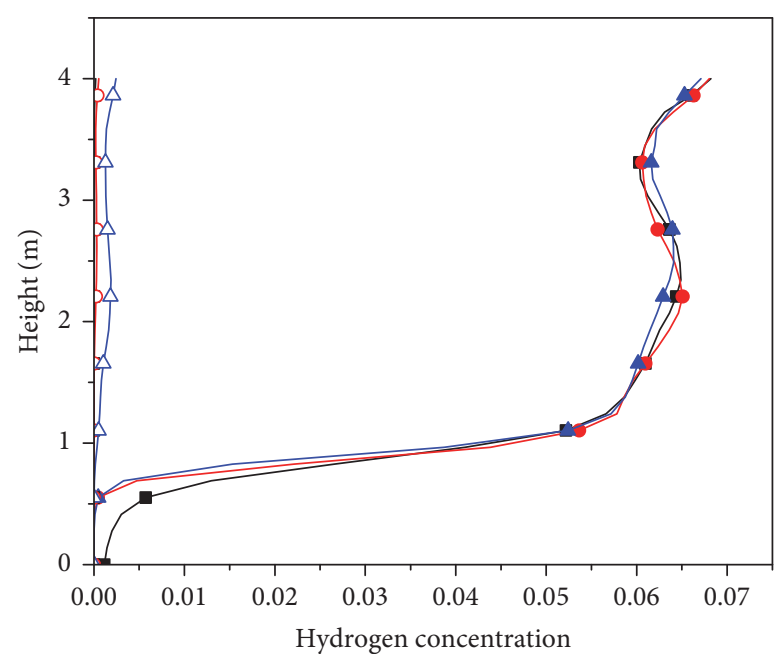

$t=1000 \mathrm{~s}$

- Case 1 , line 1

- Case 2, line 1

$\_$Case 3 , line 1 $\rightarrow-$ Case 1 , line 2

-o- Case 2, line 2

$\triangle-$ Case 3 , line 2

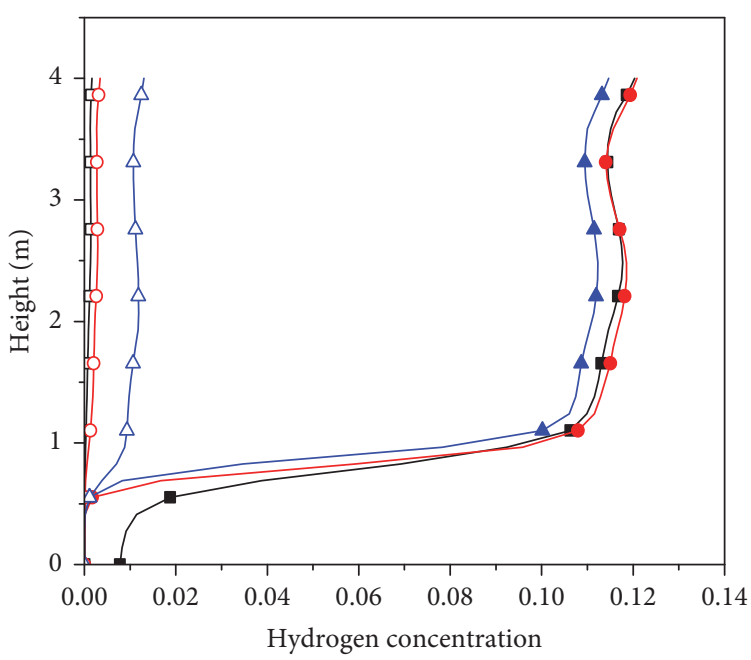

$t=2000 \mathrm{~s}$

$\rightarrow$ Case 1 , line 1

$-\square-$ Case 1 , line 2

- Case 2, line 1

-o- Case 2, line 2

$\_$Case 3 , line 1

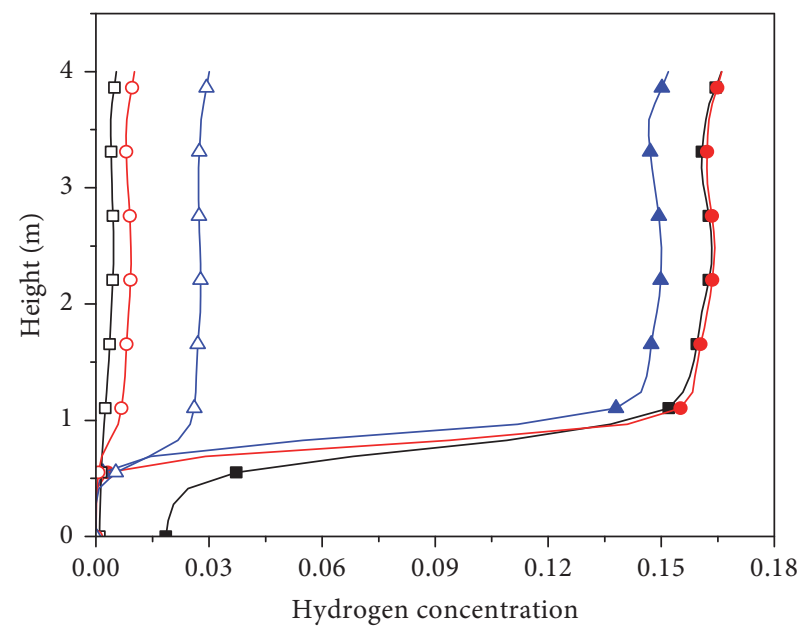

$$
t=3000 \mathrm{~s}
$$

- Case 1 , line 1

$\rightarrow-$ Case 1 , line 2

$\rightarrow$ Case 2, line 1

-o- Case 2, line 2

$\triangle-$ Case 3 , line 2

FIGURE 9: Hydrogen concentration at different positions in two compartments. 


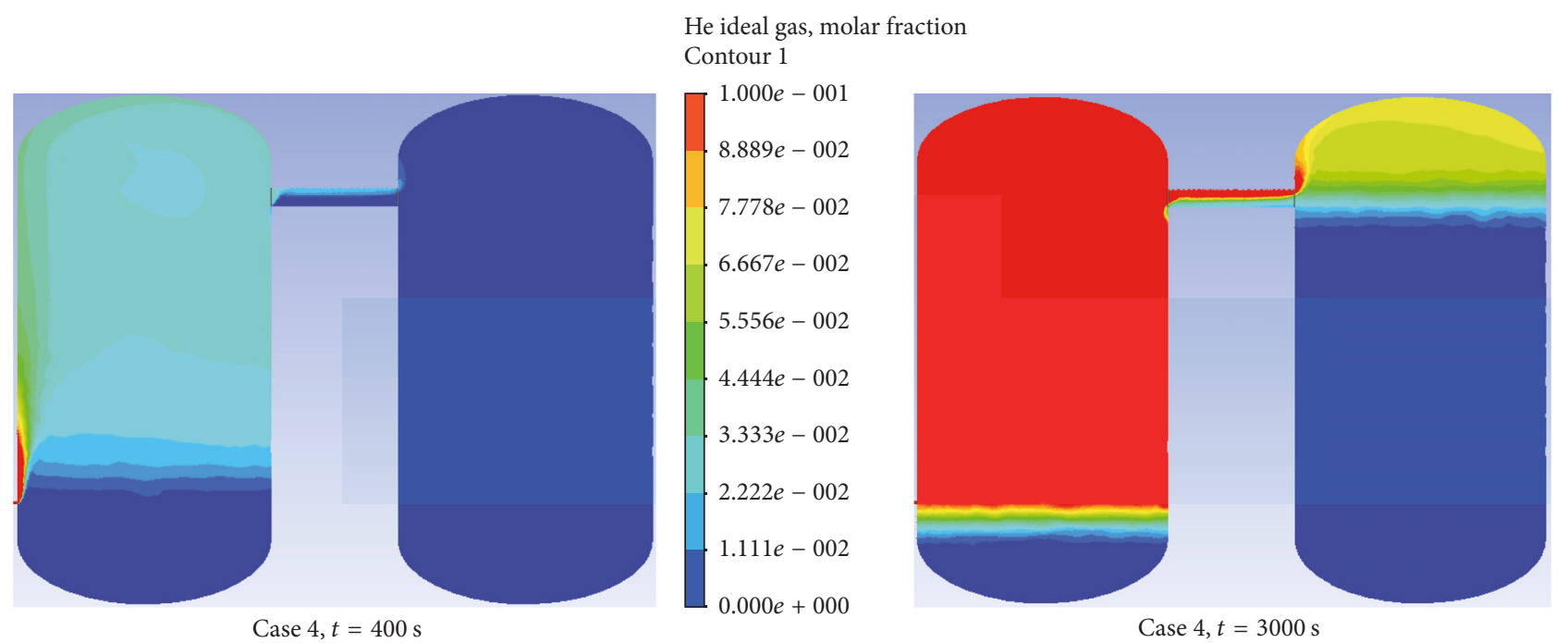

FIgURE 10: Hydrogen concentration in case 4.

at different heights in two compartments. It can be observed that the hydrogen concentration at the compartments is increased over the time with the hydrogen injection. The simulation results with cases 1,2, and 3 have the same tendency, with different hydrogen concentration at different heights in two compartments. Hydrogen concentration is relatively low in the area below the location of the IP. Comparing the results of case 1 and case 2, the values of hydrogen concentration in the upper space of two compartments are similar except for the area below the location of the IP. Due to the decrease of pipe length, hydrogen migration velocity is increased near the IP position, as a result of lower hydrogen concentration in case 2 . Compared with case 1 , hydrogen concentration in non-source term compartment is higher after the hydrogen flows into non-source term compartment in case 3 .

This shows that the diameter increase from $150 \mathrm{~mm}$ to $300 \mathrm{~mm}$ of the IP located at $800 \mathrm{~mm}$ facilitates hydrogen flow between two compartments resulting in an increase of about $13 \%$ of hydrogen molar quantity in the non-source term compartment at the end of the simulation. Decreasing the length from $1000 \mathrm{~mm}$ to $500 \mathrm{~mm}$ of the IP located at $800 \mathrm{~mm}$ can also facilitate hydrogen flow between two compartments, resulting in an increase of about $3.5 \%$ of hydrogen molar quantity in the non-source term compartment at the end of the simulation. Compared with the change of pipe diameter, the effect of pipe length change to the distribution of hydrogen in two compartments is not significant.

3.4. Changed IP Location. Figure 10 shows the hydrogen concentration distribution at $3000 \mathrm{~s}$ for case 4 . Hydrogen is accumulated in the upper space of non-source term compartment. With the effect of the pipe location at $3200 \mathrm{~mm}$, hydrogen source transports into non-source term compartment earlier compared with case 1 .

Figure 11 shows the quantitative comparison of the dispersion process in case 1 and case 4 , showing the change in hydrogen concentration at different heights in two compartments. It can be observed that hydrogen is mainly distributed in the upper space of non-source term compartment in case 4 due to higher IP location, while hydrogen flow behavior is weak in the area below the IP in non-source term compartment because of the weak diffusion process. At the time of $3000 \mathrm{~s}$, the highest hydrogen concentration in the upper space is about $7 \%$. On the contrary, for case 1 , hydrogen concentration distribution in non-source term compartment is uniform. Besides, the hydrogen concentration is lower in source term compartment in case 4 than that in case 1 , because of an increase of about $7.7 \%$ of hydrogen molar quantity flowing into the non-source term compartment when the IP location is changed from $800 \mathrm{~mm}$ to $3200 \mathrm{~mm}$ at the end of the simulation.

Higher IP location facilitates hydrogen to diffuse to the non-source term compartment from the source term compartment, so that hydrogen in the source term compartment is more easily discharged. But hydrogen may be accumulated in the upper space in the non-source term compartment if there is not enough upper space in the non-source term compartment. Lower IP location is in favor of hydrogen mixing with air in non-source term compartment due to the effect of the buoyancy.

\section{Conclusion}

This paper presents the simulation results in two compartments considering different interconnection pipe diameter, length, and location. With standard $k-\varepsilon$ turbulence model, the effects of the connecting pipe size and location on hydrogen concentration distribution are investigated.

From the comparative study, the results can be obtained:

(1) The diameter increase from $150 \mathrm{~mm}$ to $300 \mathrm{~mm}$ of the IP located at $800 \mathrm{~mm}$ facilitates hydrogen flow between two compartments resulting in an increase of about $13 \%$ of hydrogen molar quantity in the 

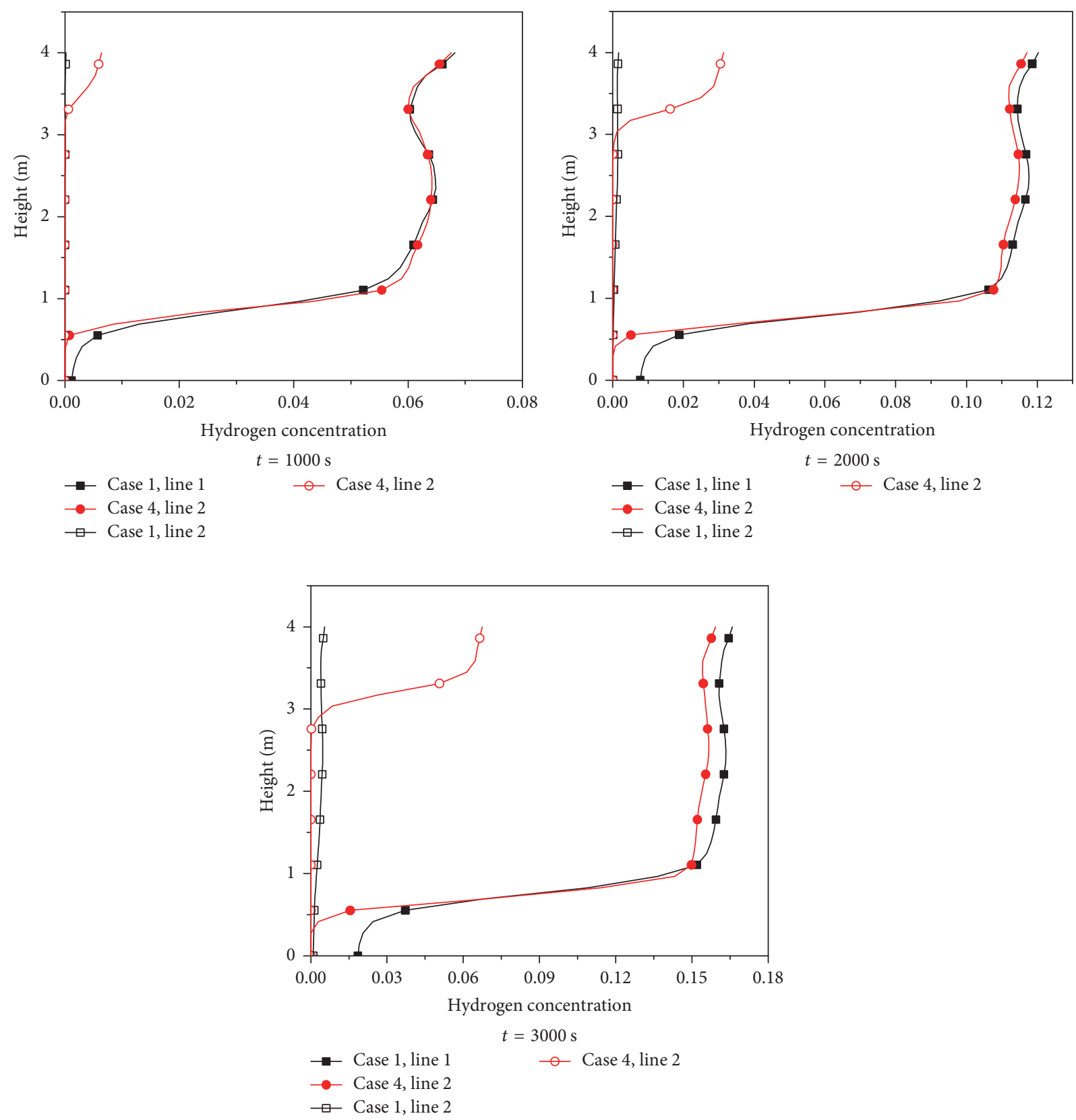

FIGURE 11: Hydrogen concentration at different positions in the compartment.

non-source term compartment at the end of the simulation.

(2) Decreasing the length from $1000 \mathrm{~mm}$ to $500 \mathrm{~mm}$ of the IP located at $800 \mathrm{~mm}$ can also facilitate hydrogen flow between two compartments, resulting in an increase of about $3.5 \%$ of hydrogen molar quantity in the non-source term compartment at the end of the simulation.

(3) Higher IP location facilitates hydrogen to diffuse to the non-source term compartment from the source term compartment, so that hydrogen in the source term compartment is more easily discharged. Lower IP location is in favor of hydrogen mixing with air in non-source term compartment due to the effect of the buoyancy.

\section{Conflicts of Interest}

The authors declare that they have no conflicts of interest.

\section{Acknowledgments}

This work was supported by National Science and Technology Major Projects (no. 2015ZX06004003-002), National Natural Science Foundation of China (no. 11675104), and Science and Technology on Reactor System Design Technology Laboratory. 


\section{References}

[1] J. Deng and X. W. Cao, "A study on evaluating a passive autocatalytic recombiner PAR-system in the PWR large-dry containment," Nuclear Engineering and Design, vol. 238, no. 10, pp. 2554-2560, 2008.

[2] M. Andreani, "Simulation of gas stratification build-up in the containment under severe accident conditions," in Proceedings of the 2014 22nd International Conference on Nuclear Engineering, ICONE 2014, Czech Republic, July 2014.

[3] U.-J. Lee and G.-C. Park, "Experimental study on hydrogen behavior at a subcompartment in the containment building," Nuclear Engineering and Design, vol. 217, no. 1-2, pp. 41-47, 2002.

[4] B. R. Sehgal, Nuclear Safety in Light Water Reactors: Severe Accident Phenomenology, Academic Press, Massachusetts, Mass, USA, 2011.

[5] Z. H. Cheng and X. W. Cao, "Numerical simulation of effect of different hydrogen production rates on hydrogen distribution within containment," Nuclear Power Engineering, vol. 28, no. 6, pp. 105-109, (In Chinese Language), 2007.

[6] D. C. Visser, M. Houkema, N. B. Siccama, and E. M. J. Komen, "Validation of a FLUENT CFD model for hydrogen distribution in a containment," Nuclear Engineering and Design, vol. 245, pp. 161-171, 2012.

[7] P. Royl, H. Rochholz, W. Breitung, J. R. Travis, and G. Necker, "Analysis of steam and hydrogen distributions with PAR mitigation in NPP containments," Nuclear Engineering and Design, vol. 202, no. 2-3, pp. 231-248, 2000.

[8] J. Kim, U. Lee, S.-W. Hong, S.-B. Kim, and H.-D. Kim, "Spray effect on the behavior of hydrogen during severe accidents by a loss-of-coolant in the APR1400 containment," International Communications in Heat and Mass Transfer, vol. 33, no. 10, pp. 1207-1216, 2006.

[9] J. Kim, S.-W. Hong, S.-B. Kim, and H.-D. Kim, "Threedimensional behaviors of the hydrogen and steam in the APR1400 containment during a hypothetical loss of feed water accident," Annals of Nuclear Energy, vol. 34, no. 12, pp. 992-1001, 2007.

[10] H. J. Allelein, K. Fischer, and J. Vendel, "International standard problem ISP-47 on containment thermal hydraulics, final report," OECD Nuclear Energy Agency (NEA), Issy-lesMoulineaux, France, 2007.

[11] D. Wang and X. W. Cao, "Numerical investigation of effect on different turbulence model for hydrogen distribution," Atomic Energy Science and Technology, vol. 50, no. 9, pp. 1622-1628, 2016.

[12] L. Q. Hou, L. L. Tong, and X. W. Cao, "Numerical research on hydrogen mixing in an experimental device," Nuclear Power Engineering, 2, pp. 146-150, 2015.

[13] D. C. Wilcox, Turbulence Modeling for CFD, DCW industries, La Canada, CA, 1998.

[14] H. Liu, K. Yuan, and X. Cao, "Numerical study on hydrogen flow behavior for different connecting pipe structures," in Proceedings of the 25th International Conference on Nuclear Engineering (ICONE-25), vol. 8, 8 pages, American Society of Mechanical Engineers (ASME), Shanghai, China, 2017. 


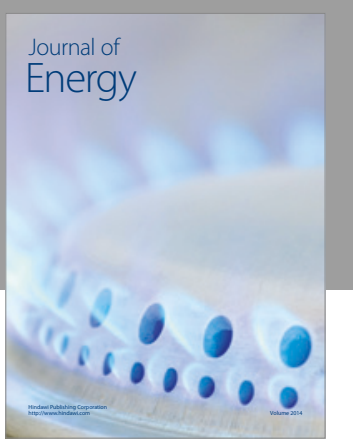

Journal of

Industrial Engineering
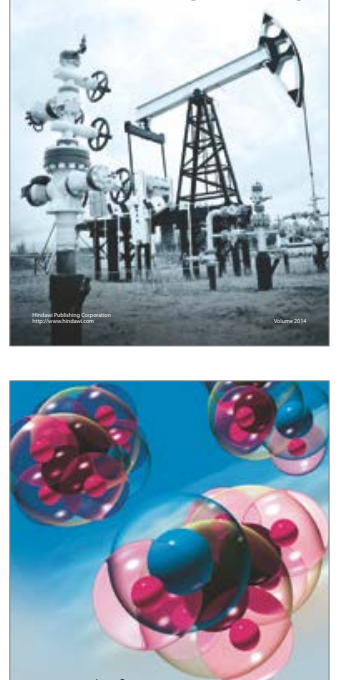

Fuels
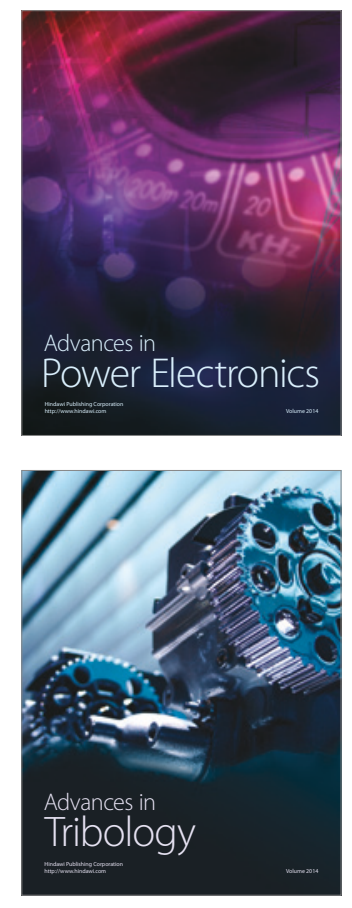
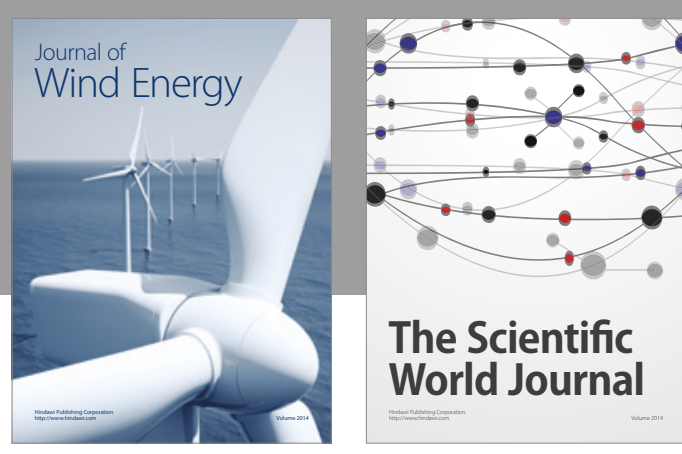

The Scientific World Journal
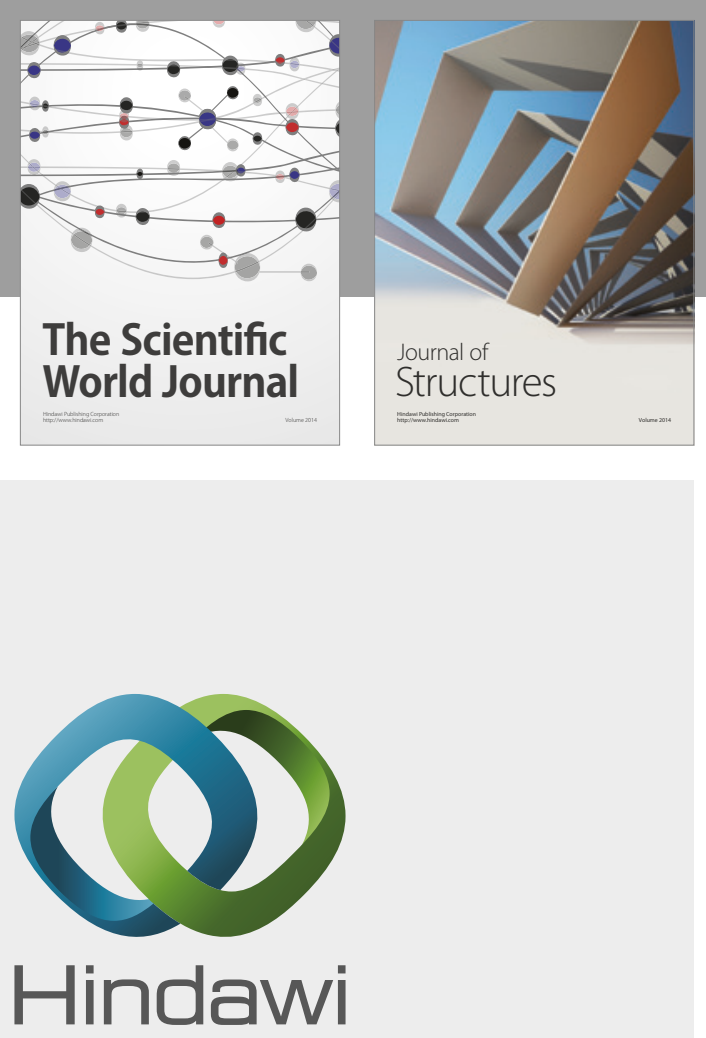

Submit your manuscripts at

https://www.hindawi.com
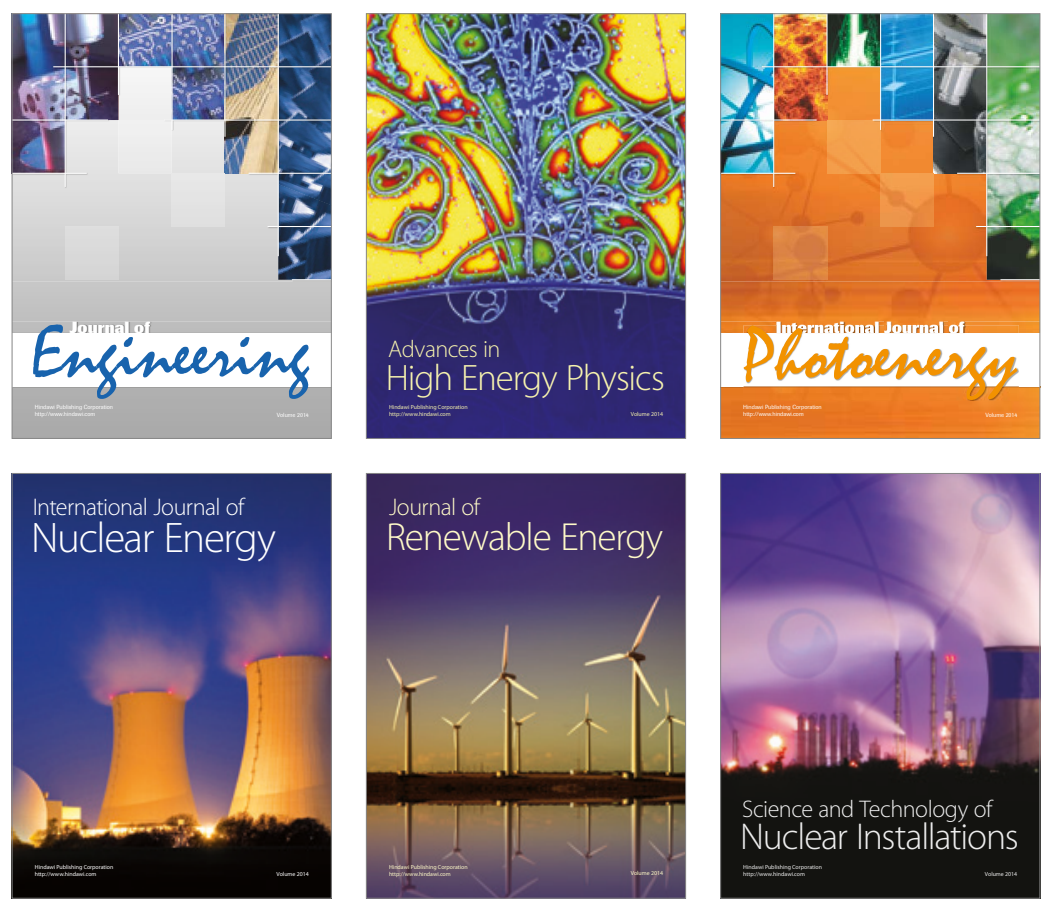

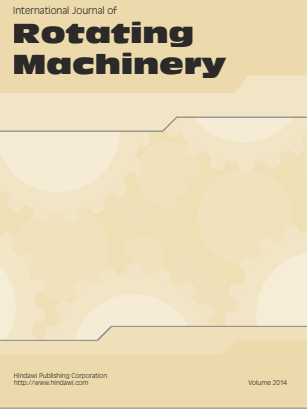

Journal of

Petroleum Engineering

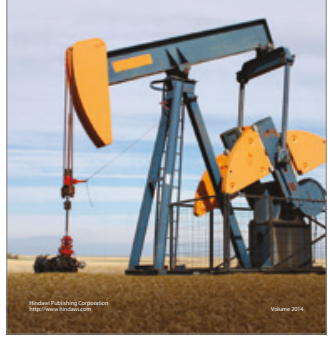

Journal of
Solar Energy
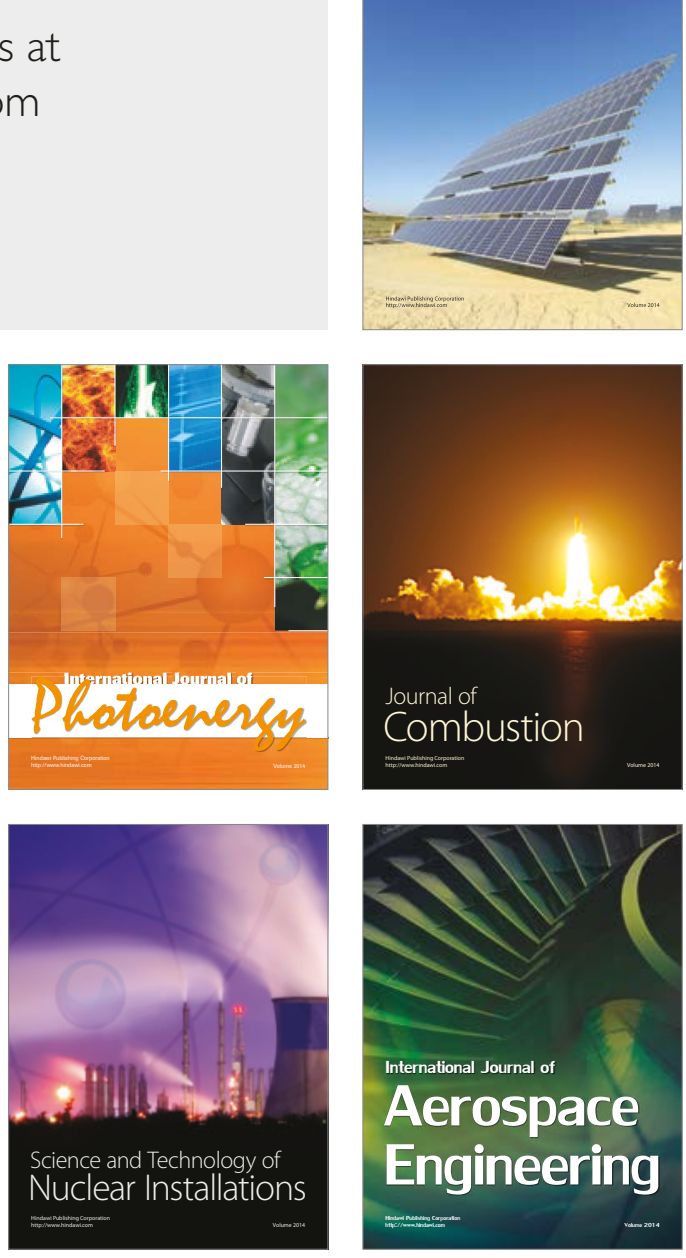